\title{
Short Rayleigh length free electron lasers
}

\author{
W. B. Colson, J. Blau, R. L. Armstead, P. P. Crooker, R. Vigil, T. Voughs, and B. W. Williams \\ Physics Department, Naval Postgraduate School, 833 Dyer Road, Monterey, California 93943, USA
}

(Received 30 November 2005; published 30 March 2006)

\begin{abstract}
Conventional free electron laser (FEL) oscillators minimize the optical mode volume around the electron beam in the undulator by making the resonator Rayleigh length about one third to one half of the undulator length. This maximizes gain and beam-mode coupling. In compact configurations of highpower infrared FELs or moderate power UV FELs, the resulting optical intensity can damage the resonator mirrors. To increase the spot size and thereby reduce the optical intensity at the mirrors below the damage threshold, a shorter Rayleigh length can be used, but the FEL interaction is significantly altered. We model this interaction using a coordinate system that expands with the rapidly diffracting optical mode from the ends of the undulator to the mirrors. Simulations show that the interaction of the strongly focused optical mode with a narrow electron beam inside the undulator distorts the optical wave front so it is no longer in the fundamental Gaussian mode. The simulations are used to study how mode distortion affects the single-pass gain in weak fields, and the steady-state extraction in strong fields.
\end{abstract}

DOI: 10.1103/PhysRevSTAB.9.030703

PACS numbers: $41.60 . \mathrm{Cr}$

\section{INTRODUCTION}

For several decades, it has been suggested that a free electron laser (FEL) oscillator can optimize the electronoptical coupling by minimizing the optical mode volume around the smaller relativistic electron beam. The origin of the idea was stated in Madey's initial paper inventing the FEL concept [1], and has lead to the common practice of designing the FEL's optical resonator so that its Rayleigh length $Z_{0}$ is about one third to one half of the undulator length $L$. That assumption is studied in this paper and it is found that there are several possible advantages to shorter Rayleigh lengths, including increased gain.

The short-Rayleigh length FEL design is an alternative that makes use of a nearly concentric resonator cavity, with a strongly focused optical mode in the center of the cavity and a large spot size at the mirrors, to reduce intensity on the mirrors for a high-power, compact FEL [2]. Scientific, industrial, and military applications of FEL oscillators can benefit from a more compact design. When the application also requires moderate to high power at infrared or shorter optical wavelengths, the conventional FEL oscillator design leads to high intensity at the mirrors and possible mirror damage.

In Madey's original paper, the FEL gain was estimated using a "filling factor" $F$ in order to describe how the smaller electron beam exchanged energy with the slightly larger optical mode. The filling factor is defined as the ratio of the electron beam area to the optical mode area. FEL single-pass gain is defined by $G=P / P_{0}-1$, where $P$ is the optical power at the end of the undulator and $P_{0}$ is the initial optical power at the beginning of the undulator. The gain is assumed to be proportional to the filling factor. A further refinement is to average the filling factor over the undulator length $L$ so that $G \propto\left(z_{0}+1 /\left(12 z_{0}\right)\right)^{-1}$. The normalized Rayleigh length is defined as $z_{0}=Z_{0} / L$ and the actual Rayleigh length is $Z_{0}=\pi W_{0}^{2} / \lambda$, where $\lambda$ is the optical wavelength and $W_{0}$ is the mode waist radius. The optimum weak-field gain is then found by minimizing the mode volume along the undulator to obtain $z_{0}=12^{-1 / 2} \approx$ 0.3 .

A couple of examples illustrate how the common practice, $z_{0} \approx 0.3 \rightarrow 0.5$, can limit an FEL's performance. Let the mirror separation of the resonator be $S=12 \mathrm{~m}$ with $10 \%$ output coupling. Typical mirrors can be damaged by high optical intensity $\sim 10 \mathrm{~kW} / \mathrm{cm}^{2}$ in the infrared and $\sim 1 \mathrm{~kW} / \mathrm{cm}^{2}$ in the UV. Assuming the electron beam and optical mode each have a radius of about $1 \mathrm{~mm}$ in the undulator, the commonly used design criteria would lead to a limitation in output power of $150 \mathrm{~W}$ in the infrared and only $3 \mathrm{~W}$ in the UV. The UV FEL is a particular problem since the longer Rayleigh length $Z_{0}$ associated with the shorter UV wavelength $\lambda$ increases mirror intensity further by decreasing the mirror spot size. Both FELs would benefit from a shorter Rayleigh length resonator in order to reduce the intensity on the mirrors and keep the system compact. The conventional estimate for gain would lead to $G \propto z_{0}$ for small Rayleigh length $z_{0} \ll 1$. However, the research presented here predicts that there is little or no loss in gain for small $z_{0} \ll 1$. The design option is illustrated in Fig. 1 below.

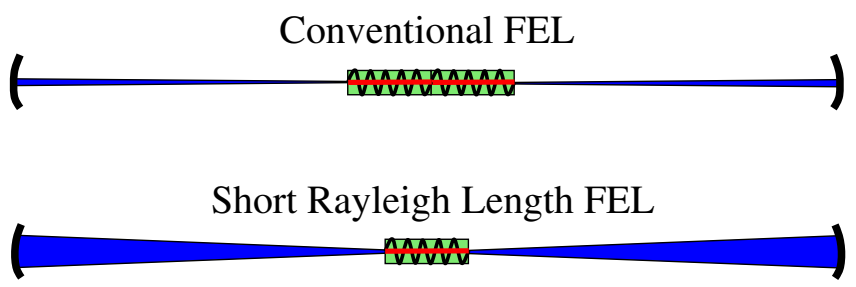

FIG. 1. (Color) Schematic comparison of the conventional and short-Rayleigh length FEL designs. 


\section{A. FEL interaction}

The FEL interaction is altered in the short-Rayleigh length FEL because the optical field amplitude and phase change significantly along the undulator [3]. The dimensionless field amplitude $a(\tau)$ and phase $\phi(\tau)$ at the mode center $r=0$ are shown in Fig. 2 and are given by

$$
\begin{gathered}
a(\tau)=a_{0}\left[1+\left(\tau-\tau_{w}\right)^{2} / z_{0}^{2}\right]^{-1 / 2}, \\
\phi(\tau)=-\arctan \left[\left(\tau-\tau_{w}\right) / z_{0}\right]
\end{gathered}
$$

where $\tau=c t / L$ is the dimensionless time of interaction along the undulator length $L, c$ is the speed of light, $a_{0}$ is the field amplitude at the mode focus, and $\tau_{w}$ is the location of the mode focus along the undulator. The dimensionless optical field amplitude is defined by $a=$ $4 \pi N e K L E / \gamma^{2} m c^{2}$, where $N$ is the number of undulator periods, $K=e B \lambda_{0} / 2 \pi m c^{2}$ is the undulator parameter, $E$ is the rms optical electric field amplitude, $B$ is the rms undulator magnetic field amplitude, $\lambda_{0}$ is the undulator period, and $e$ and $m$ are the electron charge and mass, all in cgs units. Notice from Eqs. (1) and (2) that when $z_{0}$ is small, the field amplitude and phase change rapidly along the undulator, as illustrated in Fig. 2. While the rapidly changing field would appear to be detrimental to the bunching process, it improves coupling by focusing laser light to a small waist to intensify the field strength.
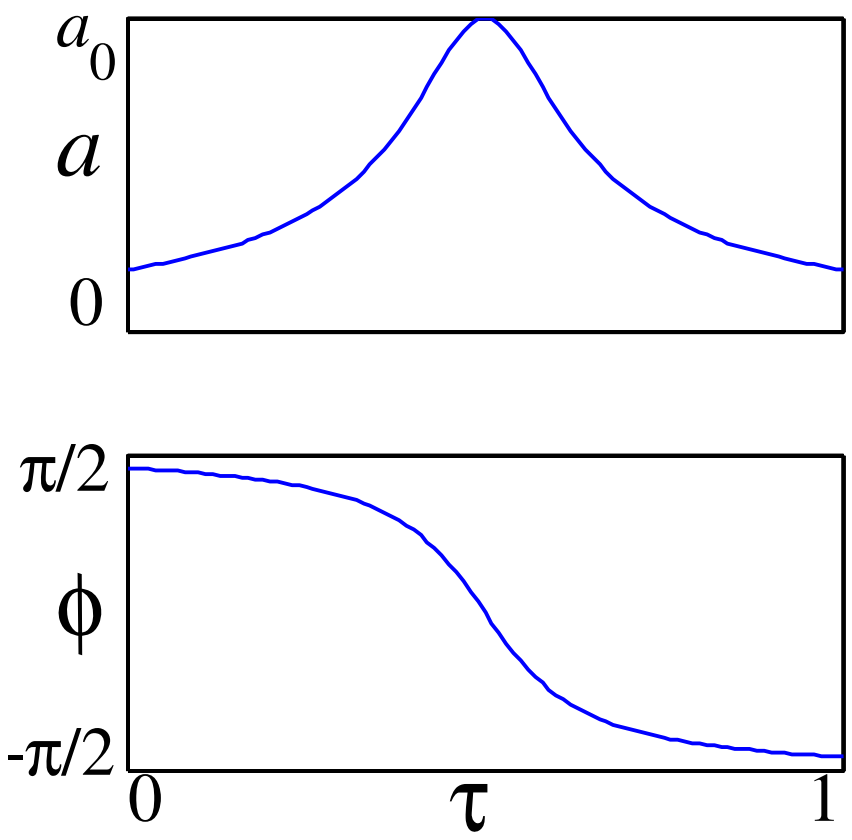

FIG. 2. (Color) Dimensionless optical field amplitude $a(\tau)$ and phase $\phi(\tau)$ versus dimensionless time $\tau=c t / L$, for a shortRayleigh length FEL, $z_{0}=0.1$. Note the rapid change in amplitude and phase near the optical waist at the center of the undulator, $\tau=\tau_{w}=0.5$.

\section{SIMULATION METHOD}

Our wave front propagation program has been described elsewhere [4]. At each time step, it uses the relativistic Lorentz force equations to determine the electron motion in the presence of the undulator and optical fields, and the paraxial wave equation to evolve the optical wave front in $(x, y, t)$ self-consistently. The simulation can follow multiple transverse optical modes as they interact with the electron beam and bounce back and forth in the optical cavity, including mirror transmission and edge losses. We typically start the simulation in weak optical fields and allow it to evolve over many passes through the cavity until the FEL reaches steady state. Graphical output shows the evolution of the electrons and the optical wave fronts, and the weak-field gain and steady-state power extraction are reported.

Our simulations use dimensionless parameters [5]. Longitudinal distances are normalized to the undulator length $L$, and transverse distances are normalized to $(L \lambda / \pi)^{1 / 2}$. Times are normalized to $L / c$, the time for a photon to travel a single pass through the undulator.

Recent improvements to our programs include a faster Fourier transform algorithm [6] and an expandingcoordinate system to follow the rapidly diffracting optical mode with a reasonable grid size $[7,8]$. We will now describe this new coordinate system in detail, then discuss how we have validated the technique and incorporated it into our FEL simulations.

\section{A. Expanding-coordinate system: Motivation}

In a compact short-Rayleigh length FEL, the area of the optical beam can be thousands of times greater at the mirrors than at the beam waist. A fixed numerical grid of sufficient resolution to represent both the narrow mode radius at the waist and the broad mode radius at the mirrors would be prohibitively large.

For example, assume a Rayleigh length of $z_{0}=Z_{0} / L=$ 0.1 and a cavity length of $s=S / L=30$, both normalized to the undulator length, $L$. Then the mode radius will expand by a factor of $\left[1+(s / 2)^{2} / z_{0}^{2}\right]^{1 / 2}=150$. If we assume 100 grid points in each dimension are needed to accurately represent the mode at the waist, then 15000 grid points are needed at the mirrors. A two-dimensional complex, double-precision array of that size requires about 4 GB of RAM, beyond the limits of many computers. Furthermore, the simulation runtime increases as the square of the number of grid points. For the typical parameters given above, we estimate it would require about $4 \mathrm{~h}$ for each pass through the optical cavity, running a three-dimensional simulation in $(x, y, t)$ on a $2 \mathrm{GHz}$ IBM G5 processor. For some sets of parameters, several hundred passes are needed to reach steady-state operation, implying that the program would take many weeks to run. Furthermore, if we wish to include a fourth dimension in 
the simulation $(z)$ to study longitudinal modes and pulse effects, we would need at least 100 slices in the $z$ direction. In addition, steady state in $4 \mathrm{D}$ requires thousands of passes. The memory requirements would then grow to 100's of GB and the simulation runtime would increase to many years.

One solution to this numerical problem is to abandon a fixed Cartesian grid, and instead use a coordinate system that expands with the diffracting optical mode. This approach is explained below for the FEL.

\section{B. Paraxial wave equation}

As usual, the equation to be solved for the complex electric field $a$ is the paraxial wave equation [9] expressed in the dimensionless coordinates described previously,

$$
a_{x x}+a_{y y}+4 i a_{\tau}=0,
$$

where a subscript in $x, y$, or $\tau$ indicates a partial derivative with respect to that variable. Equation (3) has been studied extensively and is solved reliably by Fourier transform methods $[7,8]$, except for the numerical difficulty of the expanding beam due to diffraction.

\section{Transforming the coordinates}

Consider the exact fundamental-mode solution to Eq. (3):

$$
a(x, y, \tau)=a_{0}\left(\pi z_{0} / A\right)^{1 / 2} \exp \left(-\pi r^{2} / A\right) e^{i \phi},
$$

where $r^{2} \equiv x^{2}+y^{2}$, and the dimensionless beam area is

$$
A=\pi z_{0}\left(1+\tau^{2} / z_{0}^{2}\right) .
$$

Here $a_{0}$ is the amplitude at the optical waist $(\tau=0)$ on axis $(r=0)$, the waist radius is $w_{0}=z_{0}^{1 / 2}$, and the optical phase is given by

$$
\phi(r, \tau)=-\arctan \left(\tau / z_{0}\right)+\pi r^{2} \tau /\left(A z_{0}\right) .
$$

Thus for the case where $\tau \gg z_{0}, A \approx \pi \tau^{2} / z_{0}$ and $\phi \approx$ $-\pi / 2+r^{2} / \tau$, so that

$$
a(r, \tau) \approx a_{0}\left(z_{0} / \tau\right) \exp \left(-r^{2} z_{0} / \tau^{2}\right) \exp \left(i r^{2} / \tau\right)
$$

to within a constant phase factor. It is clear from Eq. (5) that for large $\tau$ the radius of the beam expands linearly with $\tau$, which suggests that we define the new "expanding" independent variables [8]

$$
\begin{aligned}
& x^{\prime} \equiv \sqrt{z_{0}} x / \tau, \\
& y^{\prime} \equiv \sqrt{z_{0}} y / \tau,
\end{aligned}
$$

and a new dependent variable $v\left(x^{\prime}, y^{\prime}, \tau^{\prime}\right)$ such that

$$
a(x, y, \tau)=(1 / \tau) v\left(x^{\prime}, y^{\prime}, \tau^{\prime}\right) \exp \left(i r^{2} / \tau\right),
$$

where $\tau^{\prime}$ is the time in the primed (expanding) coordinates, as determined below. The choice of $\tau^{\prime}$ is made in such a way below [Eq. (17)] that the evolution of $v\left(x^{\prime}, y^{\prime}, \tau^{\prime}\right)$ is determined by the familiar paraxial wave equation in $\left(x^{\prime}, y^{\prime}, \tau^{\prime}\right)$. The phase factor $\exp \left(i r^{2} / \tau\right)$ characterizing an expanding "paraxial spherical wave" [9], as well as the spherical amplitude expansion factor $1 / \tau$, are explicitly factored out from $a$ in Eq. (10). The remaining function $v$ has to account only for the diffraction effects not contained in the solution for the Gaussian fundamental mode. In addition, the inverse dependence of $x^{\prime}$ and $y^{\prime}$ on $\tau$ means that the dimensions of the primed numerical grid decrease with increasing $\tau$, i.e., in precisely the region where the physical beam (represented on the unprimed grid $x$ and $y$ ) becomes large by diffraction.

We will now show that $v\left(x^{\prime}, y^{\prime}, \tau^{\prime}\right)$ itself does indeed satisfy exactly the same paraxial wave equation as does $a(x, y, \tau)$, Eq. (3). First, evaluate $a_{x} \equiv \partial a(x, y, \tau) / \partial x=$ $\left(v_{x^{\prime}} x_{x}^{\prime} / \tau+2 i x v / \tau^{2}\right) \exp \left(i r^{2} / \tau\right)$, by the chain rule applied to Eq. (10). But $x_{x}^{\prime} \equiv \partial x^{\prime} / \partial x=\sqrt{z_{0}} / \tau$ by Eq. (8). Thus, $a_{x}=\left(v_{x^{\prime}} \sqrt{z_{0}} / \tau^{2}+2 i x v / \tau^{2}\right) \exp \left(i r^{2} / \tau\right)$. One more derivative with respect to $x$ yields

$$
a_{x x}=\frac{e^{i r^{2} / \tau}}{\tau^{3}}\left(z_{0} v_{x^{\prime} x^{\prime}}+4 i \sqrt{z_{0}} x v_{x^{\prime}}+2 i v \tau-4 v x^{2}\right) .
$$

In the same fashion, we find that

$$
a_{y y}=\frac{e^{i r^{2} / \tau}}{\tau^{3}}\left(z_{0} v_{y^{\prime} y^{\prime}}+4 i \sqrt{z_{0}} y v_{y^{\prime}}+2 i v \tau-4 v y^{2}\right) .
$$

Now differentiating Eq. (10) with respect to $\tau$ using the chain rule again,

$$
a_{\tau}=\frac{e^{i r^{2} / \tau}}{\tau^{3}}\left(v_{x^{\prime}} x_{\tau}^{\prime} \tau^{2}+v_{y^{\prime}} y_{\tau}^{\prime} \tau^{2}+v_{\tau^{\prime}} \tau_{\tau}^{\prime} \tau^{2}-v \tau-i r^{2} v\right)
$$

Using $\quad x_{\tau}^{\prime} \equiv \partial x^{\prime} / \partial \tau=-\sqrt{z_{0}} x / \tau^{2} \quad$ and $\quad y_{\tau}^{\prime} \equiv \partial y^{\prime} / \partial \tau=$ $-\sqrt{z_{0}} y / \tau^{2}$, Eq. (13) becomes

$$
a_{\tau}=\frac{e^{i r^{2} / \tau}}{\tau^{3}}\left[-\sqrt{z_{0}}\left(x v_{x^{\prime}}+y v_{y^{\prime}}\right)+v_{\tau^{\prime}} \tau_{\tau}^{\prime} \tau^{2}-v \tau-i r^{2} v\right]
$$

Substituting Eqs. (11), (12), and (14) into Eq. (3) yields

$$
v_{x^{\prime} x^{\prime}}+v_{y^{\prime} y^{\prime}}+4 i\left(\tau^{2} \tau_{\tau}^{\prime} / z_{0}\right) v_{\tau^{\prime}}=0 .
$$

We are still free to relate $\tau$ and $\tau^{\prime}$. A convenient choice is found by imposing the condition $\tau^{2} \tau_{\tau}^{\prime} / z_{0}=1$, so that the wave equation in the primed coordinates takes the form of the paraxial wave equation,

$$
v_{x^{\prime} x^{\prime}}+v_{y^{\prime} y^{\prime}}+4 i v_{\tau^{\prime}}=0
$$

This condition is $\tau_{\tau}^{\prime} \equiv \partial \tau^{\prime} / \partial \tau=z_{0} / \tau^{2}$, which can be integrated with respect to $\tau$ to obtain

$$
\tau^{\prime}=z_{0}\left(1 / \tau_{1}-1 / \tau\right)
$$

where the constant of integration is written $z_{0} / \tau_{1}$ so that $\tau^{\prime}=0$ when $\tau=\tau_{1}$. The well-understood fast-Fouriertransform method may then be applied to Eq. (16), without 
the numerical difficulty of following a rapidly expanding wave front.

The solution for the optical field $a$ when $\tau<\tau_{1}$ is calculated in the conventional coordinates $x, y$, and $\tau$, then connected [using Eq. (10)] to the expandingcoordinate solution for $\tau \geq \tau_{1}$.

Note that a function $a(x, y, \tau)$ which expands approximately linearly with $\tau$ in the unprimed coordinate system (for example, the fundamental mode, Eq. (4), in the limit $\tau \gg z_{o}$ ) is transformed through Eq. (10) to a function $v\left(x^{\prime}, y^{\prime}, \tau^{\prime}\right)$ with nearly constant width in the expandingcoordinate system. In the simulation of an actual FEL, the interaction with the electron beam will create a mixture of modes rather than just the fundamental mode, but the transformation can handle higher-order modes as well with no modification.

The schematic representations in Figs. 3 and 4 show $|a|$ expanding as a function of $\tau$ and $|v|$ as a function of $\tau^{\prime}$ respectively, to illustrate the effect of the coordinate transformation. In an actual numerical simulation, we calculate $|a|$ in the region $\tau=0$ to $\tau=\tau_{1}$ in $(x, y, \tau)$, then switch to the primed system $\left(x^{\prime}, y^{\prime}, \tau^{\prime}\right)$ to calculate $|v|$ for $\tau>\tau_{1}$, corresponding to $\tau^{\prime}>0$. Then we apply the transformation Eq. (10) to recover $a(\tau)$ for $\tau>\tau_{1}$. The dashed lines in the diagrams remind us of the relative size of the integration steps in the primed and unprimed systems.

In terms of the numerical integration using the expanding coordinates, constant time steps $\Delta \tau^{\prime}$ correspond to time steps in the unprimed coordinates which increase quadratically with $\tau$, so that $\Delta \tau=\tau^{2} \Delta \tau^{\prime} / z_{0}$, as required by Eq. (17). These increasing time steps are beneficial in propagating out to mirrors far away from the end of the undulator.

\section{Validation}

We first used the expanding-coordinate method to study free-space diffraction of a fundamental mode, and validated the results by comparison to Gaussian beam theory

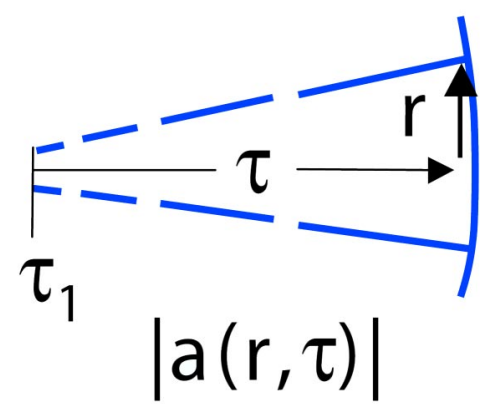

FIG. 3. (Color) Contours of constant optical field amplitude $|a|$ as a function of Cartesian coordinates $(r, \tau)$ for free-space diffraction of a nearly spherical wave front, for $\tau_{1} \gg z_{0}$. The dashed lines indicate the increasing size of the integration steps in the unprimed coordinate system.

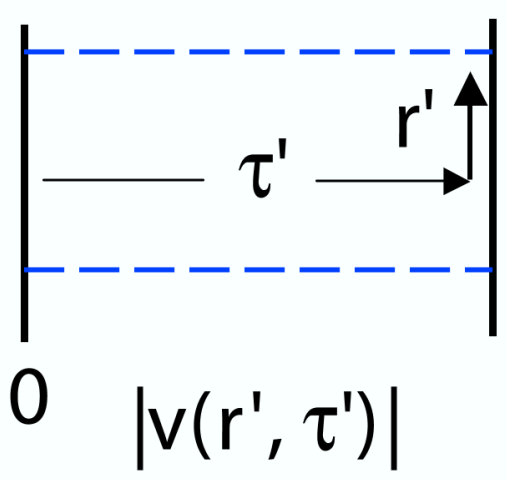

FIG. 4. (Color) Contours of constant virtual field amplitude $|v|$ as a function of expanding coordinates $\left(r^{\prime}, \tau^{\prime}\right)$ for free-space diffraction of a spherical wave front. The dashed lines indicate the constant size of the integration steps in the primed coordinate system. Also notice that a spherical wave maintains a nearly constant radius in this coordinate system.

[10]. Then we used expanding coordinates to model the evolution of optical wave fronts in a cold cavity, with mirrors of various curvature, including the effects of misaligned mirrors. The results were compared to ray-tracing algorithms, showing good agreement over many orders of magnitude [11]. We have now incorporated this expandingcoordinate method into our FEL simulations. Inside the undulator, we use a normal Cartesian coordinate system. From the ends of the undulator to the mirrors, we use the expanding-coordinate system described above.

\section{SIMULATION RESULTS: WEAK OPTICAL FIELDS}

First we use our simulations to study FEL behavior in weak optical fields, $|a|<\pi$. In particular, we look at the single-pass gain as the Rayleigh length is varied. As an example, consider an FEL having an undulator with $N=$ 22 periods, total length $L=52 \mathrm{~cm}$, rms undulator parameter $K=1$, and period $\lambda_{0}=2.36 \mathrm{~cm}$. An FEL with a shortRayleigh length requires a short undulator length so that the expanding mode does not scrape the undulator magnets. In this case the undulator gap $g=1 \mathrm{~cm}$ and there is no significant scraping of the focused electron beam or optical mode. At the end of the undulator the optical mode radius is $W_{u} \approx\left(\lambda L / 4 \pi z_{0}\right)^{1 / 2} \approx 0.2 z_{0}^{-1 / 2} \mathrm{~mm}$. In order to keep $W_{u}<0.1 \mathrm{~g}=1 \mathrm{~mm}$ to avoid even the slightest scraping, we keep $z_{0}>0.05$. The electron micropulse has peak current $\hat{I}=400 \mathrm{~A}$ and energy $E_{b}=80 \mathrm{MeV}$, corresponding to a Lorentz factor $\gamma=E_{b} / m c^{2} \approx 157$. The electron beam is focused to a narrow waist radius $r_{b}=0.06 \mathrm{~mm}$. The FEL resonance condition defines the optical wavelength as $\lambda \approx \lambda_{0}\left(1+K^{2}\right) / 2 \gamma^{2}=1 \mu \mathrm{m}$ in a resonator with mirror separation $S=18 \mathrm{~m}$ and $25 \%$ output coupling.

Figure 5 shows simulation results for this FEL, varying the dimensionless Rayleigh length over the range $z_{0}=$ 


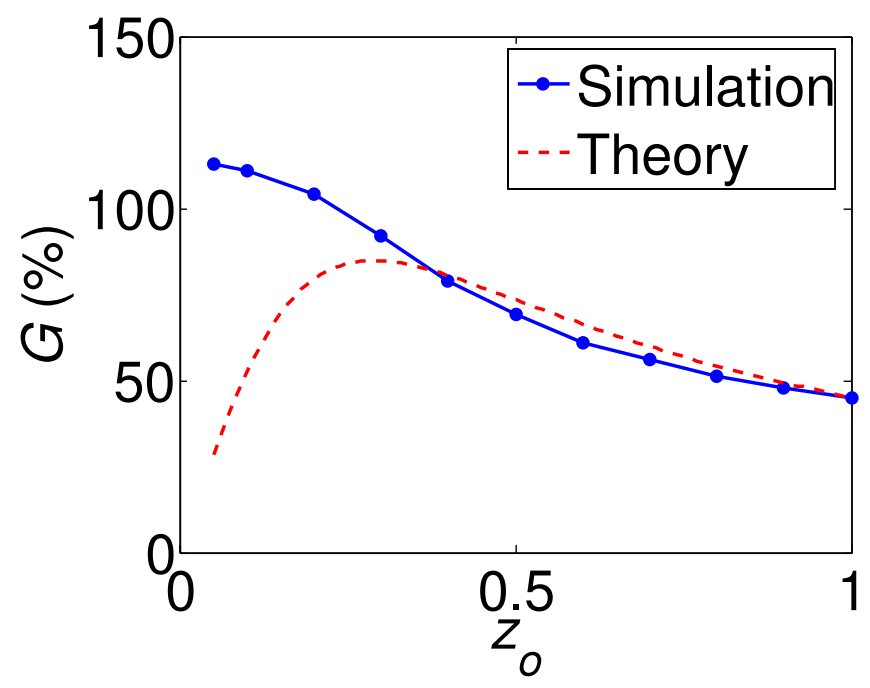

FIG. 5. (Color) Simulation results for FEL weak-field gain as a function of normalized Rayleigh length $z_{0}$ (solid blue line). The results are compared to a simple theory (dashed red line), which assumes the optical field is in the fundamental mode. The large disagreement between the simple theory and simulations for small $z_{0}$ is due to mode distortion.

$0.05 \rightarrow 1$. Also shown on this graph is the theoretical gain, which assumes the optical field is in the fundamental mode, and the gain is proportional to the filling factor. The most dramatic result is that in the simulations, the gain does not decrease like $G \propto z_{0}$ for small $z_{0}$; in fact, the gain continues to increase as $z_{0}$ is reduced below 0.3. This is due to optical mode distortion that is allowed to occur self-consistently in the simulations, but not in the simple theory, which assumes the laser operates only in the fundamental mode. The single-pass gain is fairly large for this example $(G \approx 50 \rightarrow 100 \%)$, but the trend shown here has been confirmed for much smaller gain as well. In fact, only very low gain FELs $(G \lesssim 2 \%)$ show decreasing gain at small Rayleigh lengths.

The observed mode distortion is not large, but sufficient to significantly increase the gain when the Rayleigh length is small. In these cases, the electron beam has been focused to a waist size of $r_{b} \approx 0.06 \mathrm{~mm}$ so that it remains inside the focused optical mode waist. The normalized emittance required for this case is $\epsilon_{n}=3 \mathrm{~mm}$-mrad, but larger emittances we have explored show that there is no significant decrease in gain at small Rayleigh length when mode distortion is allowed.

\section{SIMULATION RESULTS: STRONG OPTICAL FIELDS}

Now we use our simulations to study the FEL behavior when it reaches steady-state saturation in strong optical fields, $|a| \gg \pi$. In particular, we look at the single-pass extraction, defined as the output optical power divided by the input electron beam power, as various FEL parameters are modified. We simulate the same FEL described in the previous section. The dimensionless current density is given by

$$
j=\frac{8 N(e \pi K L)^{2} \rho_{e}}{\gamma^{3} m c^{2}}
$$

where $\rho_{e}$ is the electron particle density, proportional to the peak beam current $\hat{I}$. For the current set of parameters, $j=$ 99. The dimensionless electron beam radius is $\sigma=$ $r_{b} /(L \lambda / \pi)^{1 / 2}=0.15$ and the dimensionless Rayleigh length is $z_{0}=Z_{0} / L=0.12$. The dimensionless cavity length is $s=S / L=35$.

First we ran a series of simulations varying the Rayleigh length from $z_{0}=0.05 \rightarrow 1$, normalized to the undulator length $L$. The results for extraction versus Rayleigh length are shown in Fig. 6. Extraction $\eta$ is defined as the fraction of electron beam power converted to optical power on a single pass through the undulator. Also shown on that graph is the intensity on the mirrors versus Rayleigh length. The results confirm that a short-Rayleigh length FEL can maintain good extraction while reducing optical intensity on the mirrors.

Figure 7 shows the steady-state extraction from many simulations as the current density $j$ is varied while emittance is held constant. A theoretical curve, discussed below, is also shown for reference. The Rayleigh length is kept fixed at $z_{0}=0.12$. Since the weak-field gain is proportional to $j$, there is a minimum threshold value of $j \approx$ 20 below which there is no extraction, because the cavity losses exceed the gain. For larger values of $j$, the power will grow over each pass until the laser reaches saturation in strong optical fields. Basic FEL theory [5] predicts that for high gain, $j \gg 1$, the extraction should grow as

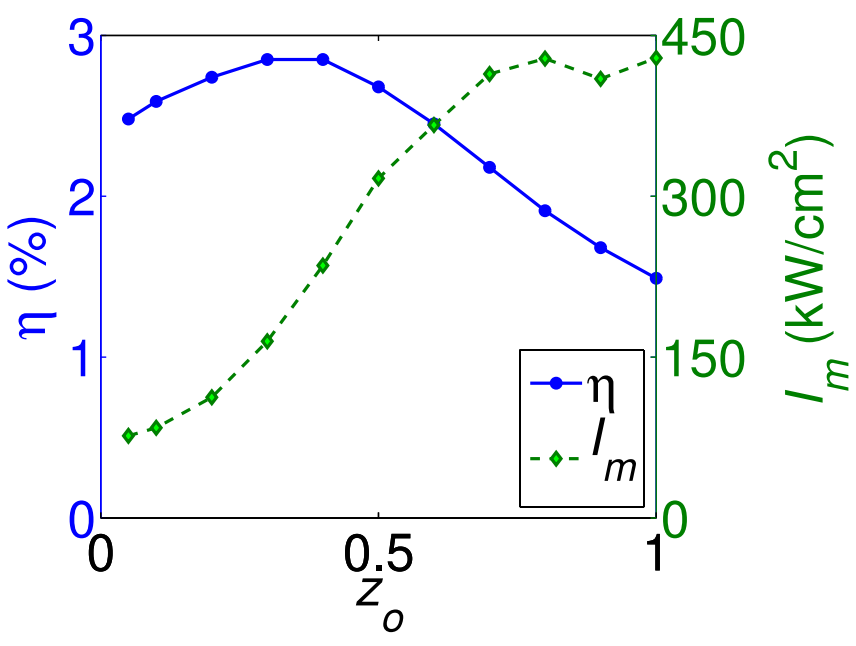

FIG. 6. (Color) Simulation results for extraction $\eta$ versus normalized Rayleigh length $z_{0}$ (solid blue line). The optimum value is $z_{0} \approx 0.3$, in agreement with the simple theory, but good extraction is maintained for smaller values of $z_{0}$. Also plotted is the intensity on the mirrors (dashed green line); the intensity decreases dramatically as the Rayleigh length is reduced. 


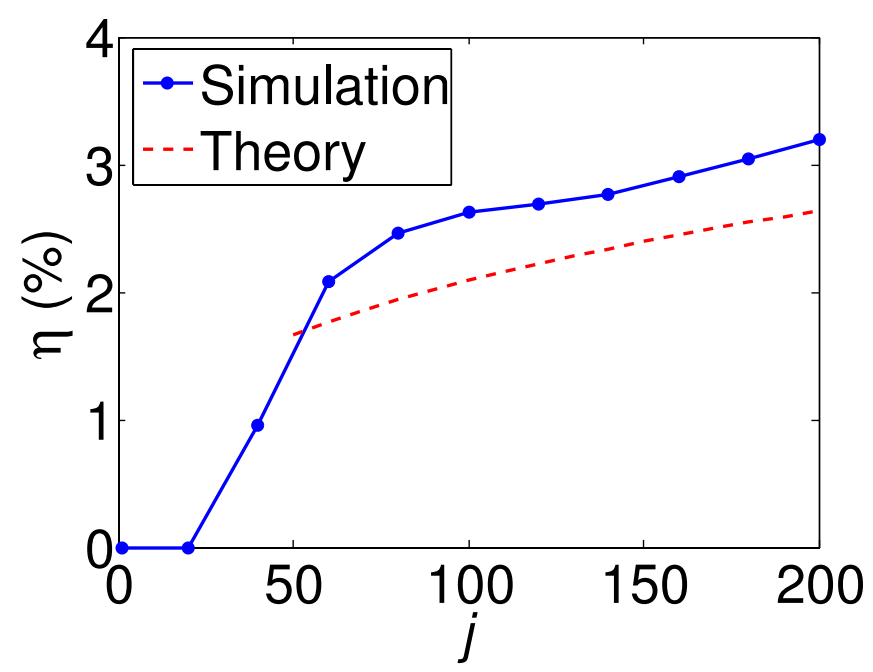

FIG. 7. (Color) Extraction $\eta$ versus dimensionless current density $j$, for a series of simulations (solid blue line), compared to theory (dashed red line). The shape of the simulation curve agrees well with the theory curve, but there is an offset, probably due to mode distortion, which the theory does not include.

$$
\eta \approx \frac{(j / 2)^{1 / 3}}{8 N}
$$

Equation (19) is plotted as a dashed red line in Fig. 7. The similar slope of the two curves confirms the $j^{1 / 3}$ dependence, but the theory curve is slightly below the simulation curve. However, Eq. (19) is only an approximation, and does not include the effects of mode distortion.

Figure 8 shows the steady-state optical wave front profile at the output mirror for several values of $j$. For moderate current, $j=40$, the laser appears to be operating close to the fundamental mode, and for high current, $j=$ 200 , the mode is clearly distorted.

Figure 9 shows the results of many simulations as the electron beam radius $\sigma$ is varied. The beam angular spread is also varied to keep the emittance constant. The simulations predict that the optimal beam radius is at $\sigma \approx 0.1$. For small beam radii, the corresponding large angular spread reduces overlap with the optical beam over the length of the undulator, hence lowering extraction. For large beam radii, many electrons are outside the strongly

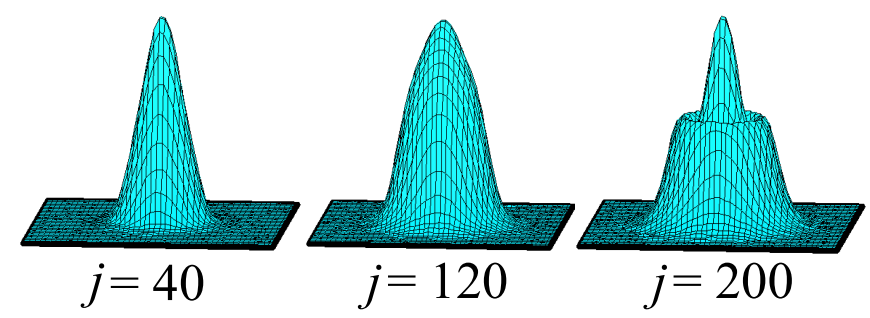

FIG. 8. (Color) Optical field amplitude $|a(x, y)|$ at the output mirror for several values of current density $j$. Mode distortion is more apparent as the current density is increased.

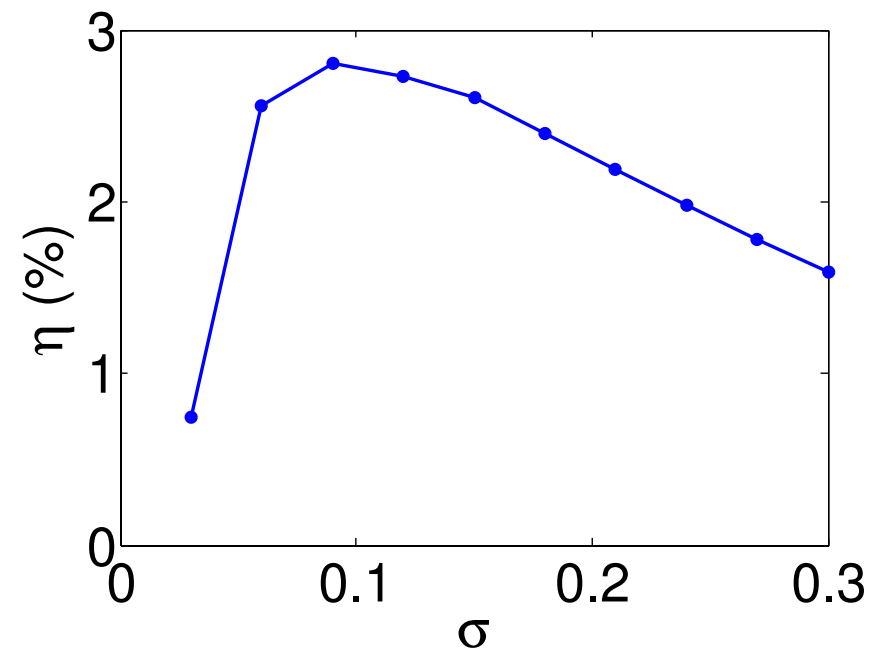

FIG. 9. (Color) Simulation results for extraction $\eta$ versus normalized electron beam radius $\sigma$, showing an optimal beam radius at $\sigma \approx 0.1$.

focused optical beam at the waist, again reducing extraction. Figure 10 illustrates these two extreme cases.

Finally, we look at the effects of varying the number of undulator periods $N$, keeping the undulator period $\lambda_{0}$ constant. The simulation results are plotted in Fig. 11. Note that changing $N$ affects many other dimensionless parameters; for example, the electron current density $j \propto$ $N^{3}$. Consequently, if there are too few periods, the gain will be below threshold, and the simulation results indeed show that there is no extraction for $N<12$, corresponding to $j<$
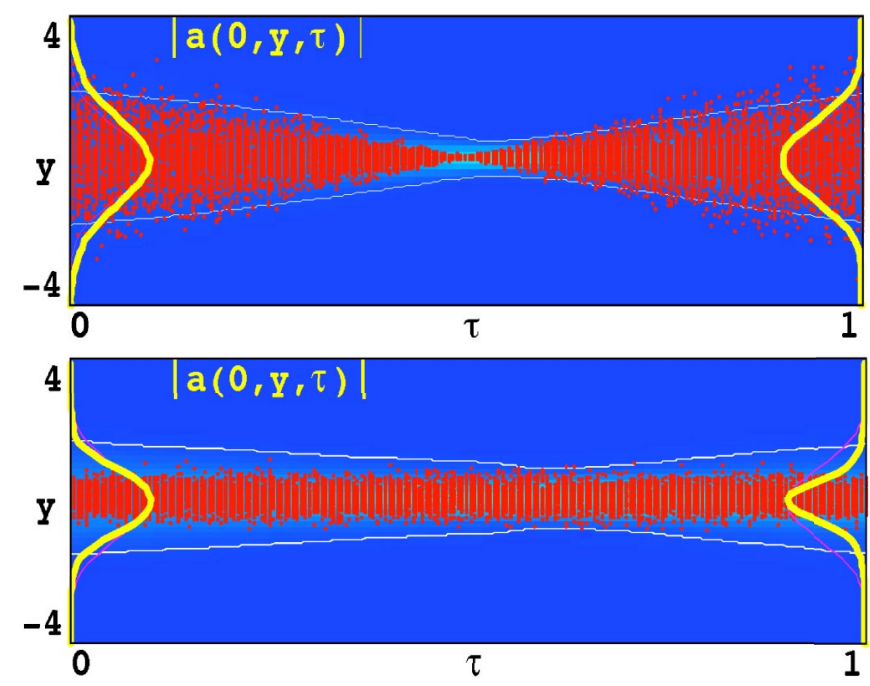

FIG. 10. (Color) A cross section of the optical mode amplitude, $|a(y, \tau)|$, over a single pass through the undulator, from $\tau=0 \rightarrow$ 1 , for a narrow electron beam $(\sigma=0.03$, top) and a broad electron beam ( $\sigma=0.3$, bottom). The red dots represent sample electrons. The white contour lines indicate $5 \%$ of the peak optical amplitude. The yellow curves on the left and right show the optical mode profile at the beginning and end of the undulator, respectively. 


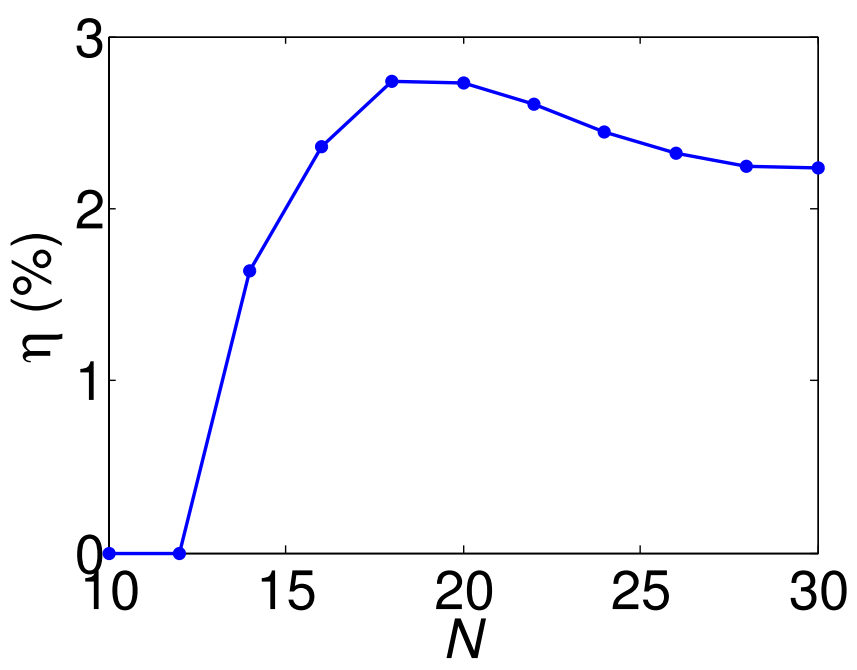

FIG. 11. (Color) Simulation results for extraction $\eta$ versus number of undulator periods $N$. For the given set of parameters, the optimal number of periods is $N \approx 18$, much shorter than a typical FEL undulator.

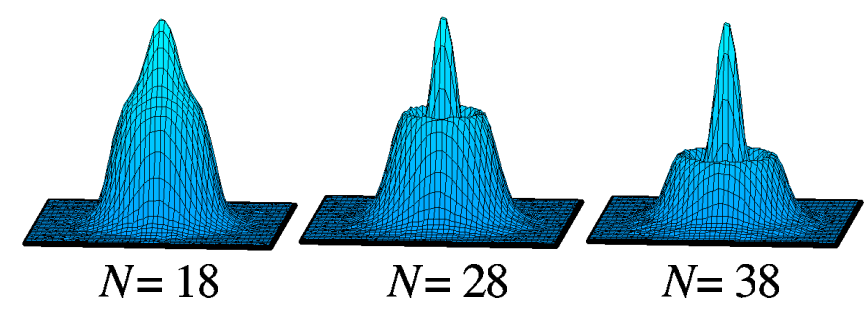

FIG. 12. (Color) Optical field amplitude $|a(x, y)|$ at the output mirror for various number of undulator periods $N$. Increasing the number of periods, in addition to reducing the extraction, also distorts the optical mode away from the fundamental.

20. Above that, the extraction increases rapidly, until $N \approx$ 18. For larger values of $N$, even though $j$ continues to increase, the extraction drops off. The optical wave fronts in Fig. 12 predict the development of mode distortion as $N$ increases.

We also varied the electron beam focus point from $\tau_{\beta}=$ $0 \rightarrow 1$, normalized to the undulator length $L$. The simulation results (not shown here) gave a fairly constant extraction over the entire range, predicting that the shortRayleigh length FEL should be fairly insensitive to fluctuations in the electron beam focal point. The optical mode profiles also did not change much over this range.

\section{CONCLUSION}

The results of this work show that it is feasible to build an FEL with a short-Rayleigh length optical cavity, which is a necessary component of a compact, high-power FEL oscillator. We have developed simulations using an expanding-coordinate system to follow the evolution of the rapidly diffracting optical fields in a short-Rayleigh length FEL. We have used these simulations to show that for normalized Rayleigh length $z_{0}<0.3$, the gain is increased significantly above the prediction of the simple theory. The simulations indicate good power extraction with reduced mirror intensity as the Rayleigh length is decreased. The simulations also show that the output wave front is increasingly distorted from the fundamental mode as the current density and/or number of undulator periods is increased. The mode distortion tends to enhance the FEL gain and extraction. We believe that this mode distortion is responsible for the deviations from the simple theory, which assumes only a single Gaussian mode.

\section{ACKNOWLEDGMENTS}

The authors are grateful for the support from NAVSEA, ONR, and the JTO.

[1] J. M. J. Madey, J. Appl. Phys. 42, 1906 (1971).

[2] D. W. Small, R. K. Wong, W. B. Colson, R. L. Armstead, Nucl. Instrum. Methods Phys. Res., Sect. A 393, 262 (1997).

[3] W. B. Colson, J. Blau, and R. L. Armstead, Nucl. Instrum. Methods Phys. Res., Sect. A 507, 48 (2003).

[4] J. Blau, Ph.D. dissertation, Naval Postgraduate School, 2002, http://handle.dtic.mil/100.2/ADA401739

[5] W. B. Colson, in Laser Handbook, edited by W. B. Colson, C. Pellegrini, and A. Renieri (North Holland, Amsterdam, 1990), Vol. 6, Chap. 5.

[6] M. Frigo and S. G. Johnson, Proc. IEEE 93, 216 (2005).

[7] E. A. Sziklas and A.E. Siegman, Proc. IEEE 62, 410 (1974).

[8] E. A. Sziklas and A.E. Siegman, Appl. Opt. 14, 1874 (1975).

[9] A.E. Siegman, Lasers (University Science Books, Sausalito, CA, 1986).

[10] R. L. Armstead, J. Blau, and W. B. Colson, in Proceedings of the 2004 Free Electron Laser Conference, Trieste, Italy, 2004 (Sincrotrone Trieste, Trieste, Italy, 2004), p. 75.

[11] P. P. Crooker, J. Blau, and W. B. Colson, Phys. Rev. ST Accel. Beams 8, 040703 (2005). 